\title{
Role of screening agar plates for in vitro susceptibility testing of Helicobacter pylori in a routine laboratory setting
}

\author{
V J Warburton-Timms, C A M McNulty
}

\begin{abstract}
Background-Resistance of Helicobacter pylor $i$ to the more frequently used antibiotics (metronidazole and clarithromycin) reduces eradication rates even with triple treatment. Determining the antibiogram profile of $H$ pylori can take up to 14 days and delays appropriate treatment.

Aims-To determine the role of screening agar plates for more rapid in vitro susceptibility of $H$ pylori to metronidazole, amoxicillin, and clarithromycin.

Methods-Routine gastric biopsy specimens from 507 dyspeptic patients were inoculated on to $10 \%$ lysed blood agar plates containing metronidazole $(8 \mu \mathrm{g} /$ $\mathrm{ml})$, clarithromycin $(2 \mu \mathrm{g} / \mathrm{ml})$, or amoxicillin $(0.5 \mu \mathrm{g} / \mathrm{ml})$. The minimum inhibitory concentration (MIC) of the 90 isolates was determined using the $\mathrm{E}$ test. Results-Metronidazole resistance was detected in 28 of 90 isolates by $E$ test and nine of 98 by screening agar. The screening agar detected none of the four clarithromycin resistant isolates detected by the E test.

Conclusions-The screening agar method is not sufficiently sensitive to be used alone.

(F Clin Pathol 2001;54:408-411)
\end{abstract}

Keywords: Helicobacter pylori; metronidazole; clarithromycin; susceptibility testing

The bacterium Helicobacter pylori infects about $40 \%$ of the population and is responsible for the development of duodenal ulcer disease. ${ }^{1}$ The infection also has a causative role in gastric ulcer disease, non-ulcer dyspepsia, and gastric adenocarcinoma, and is classed as a type 1 carcinogen by the World Health Organisation. The treatment of $H$ pylori involves the use of a proton pump inhibitor and two or three antibiotics, usually metronidazole, amoxicillin, or clarithromycin. Treatment efficacy can drop from $86 \%$ to $58 \%$ if the infecting strain is resistant to metronidazole or clarithromycin. ${ }^{2}$ Metronidazole and clarithromycin resistant $H$ pylori strains are increasing. The prevalence of primary metronidazole resistance varies from $7 \%$ to $90 \%$, with the developing world having the highest prevalence. Primary resistance to clarithromycin is between $4 \%$ and $7 \%$ and rising, ${ }^{4}$ and recently amoxicillin resistance has been reported. ${ }^{5}$ Previous antibiotic use of these agents increases the likelihood of a resistant $H$ pylori infection. ${ }^{6}$
Detecting antibiotic resistant isolates by traditional methods (disc, epsilometer (E) test, or agar dilution) takes between 10 and 14 days; therefore, empirical treatment is often started before sensitivity results are known. Primary sensitivity testing on antibiotic containing medium could reduce reporting time to three to five days. Preliminary data on this screening agar method reported by Glupczynski et al suggest that $84 \%$ of metronidazole resistant isolates can be detected. ${ }^{7}$ The aim of our study was to confirm the usefulness of this screening agar method in detecting resistance to metronidazole, clarithromycin, and amoxicillin. Our study was run in parallel with a multicentre European study determining the prevalence of $H$ pylori resistance across Europe by the E test. ${ }^{8}$ The E test was used as the gold standard because it correlates well with agar dilution, allowing Chaves et al to use it as a reference method. ${ }^{9}$

\section{Methods}

PATIENTS

Five hundred and seven consecutive routine biopsy samples from patients (>18 years) attending for upper gastrointestinal endoscopy at Gloucestershire Royal NHS Trust endoscopy clinic over a six month period were used in our study. Patients who had been treated previously with nitroimidazoles and/or macrolides were excluded.

ISOLATION OF H PYLORI AND INOCULATION OF SCREENING AGAR

Biopsy specimens for culture were sent in normal saline to the Gloucester Public Health Laboratory. Biopsy specimens were inoculated on to a chocolate agar plate (Columbia agar base CM331; Oxoid, Basingstoke, UK), three screening agar plates, and an $H$ pylori selective agar (Dent; Oxoid). The biopsy specimens were not crushed before inoculation because a large series has shown that this is not warranted. ${ }^{10}$ The three screening agar plates contained metronidazole $(8 \mu \mathrm{g} / \mathrm{ml})$, amoxicillin $(0.5 \mu \mathrm{g} / \mathrm{ml})$, and clarithromycin $(2 \mu \mathrm{g} / \mathrm{ml})$ with $10 \%$ lysed horse blood and Columbia agar base (CM331; Oxoid). ${ }^{7}$ To control for growth, the biopsy specimen was smeared on the screening agar plates in between the two culture plates. There were only four occasions when there was no growth on the $H$ pylori selective agar when $H$ pylori had grown on chocolate agar; in three of these cases growth on chocolate agar was scanty. Helicobacter pylori type strain NCTC11637 and a recently isolated strain 

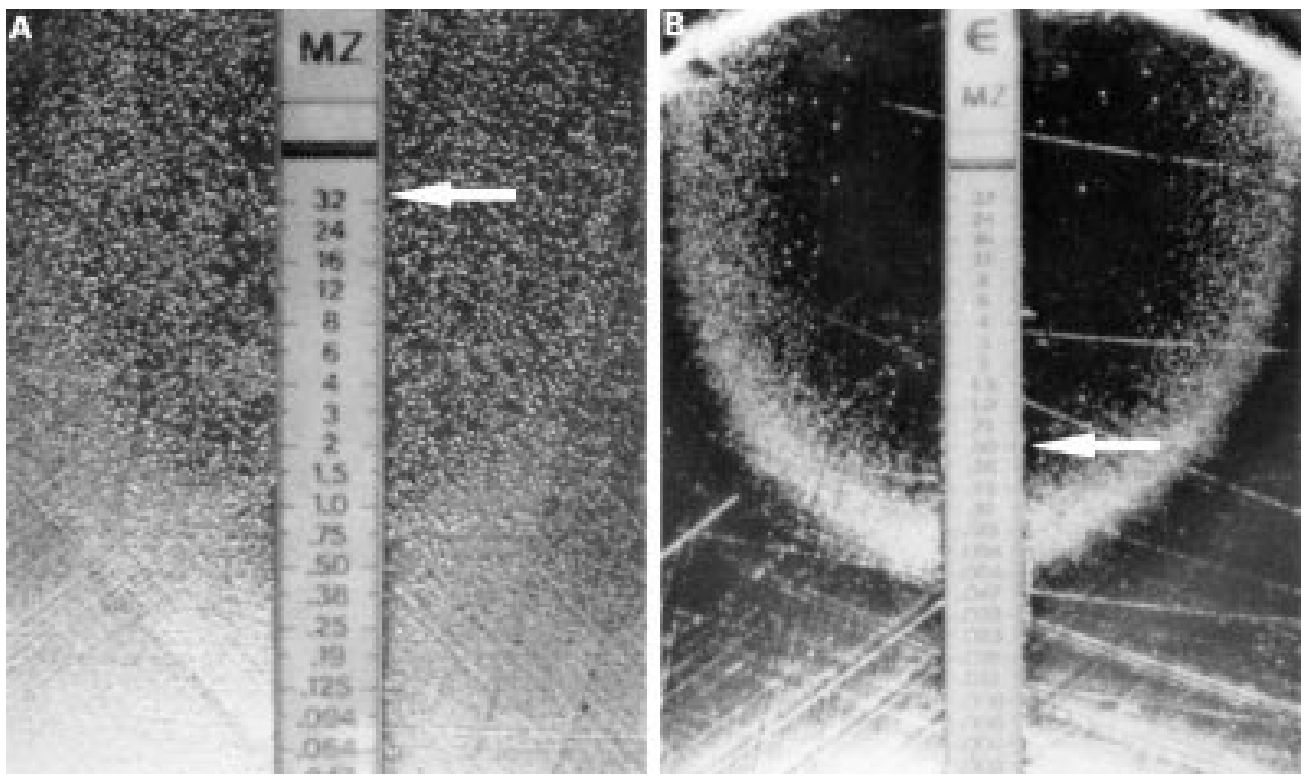

Figure 1 Reading guide for $E$ tests. (A) Colonies of a metronidazole resistant subpopulation in the ellipse minimum inhibitory concentration (MIC) > 32; (B) trailing of microcolonies at the end point MIC $0.5 \mu \mathrm{g} / \mathrm{ml}$.

were incubated with the isolates to control for changes in atmospheric conditions. Plates were incubated in a microaerobic atmosphere $(6 \%$ $\mathrm{O}_{2}, 10 \% \mathrm{CO}_{2}$ ), obtained by evacuation of air to $500 \mathrm{~mm} \mathrm{Hg}$ and replacement with the appropriate gas mixture $\left(10 \% \mathrm{H}_{2}, 10 \% \mathrm{CO}_{2}, 80 \%\right.$ $\mathrm{N}_{2}$ ), at $37^{\circ} \mathrm{C}$ for five to seven days. After five days the plates were checked for growth. If there was no growth the plates were incubated for a further two days. All agar plates were scored as scanty, moderate, or profuse growth with $H$ pylori (confirmed by urease, catalase, and Gram film), or no growth.

E TEST

The method agreed by the European $H$ pylori study group was used. ${ }^{8}$ Primary isolates of $H$ pylori were subcultured from the nonselective or Dents primary culture plates for the E test. A 48 hour fresh $H$ pylori culture was harvested into $5 \mathrm{ml}$ peptone water tubes. The inoculum density was adjusted to four on the MacFarland scale (approximately $10^{8}$ colony forming units $/ \mathrm{ml}$ ). Mueller Hinton agar (CM337; Oxoid) with 10\% horse blood was used and the $\mathrm{pH}$ of the medium was adjusted to between 7.2 and 7.4. All plates were warmed at room temperature before use. Three plates were inoculated for each strain, one plate for each $\mathrm{E}$ test strip. A lawn of inoculum was produced with the suspension using a sterile swab. Plates were allowed to dry for 10 minutes before applying the $\mathrm{E}$ test strips. The plates were incubated at $37^{\circ} \mathrm{C}$ in a microaerobic atmosphere $\left(6 \% \mathrm{O}_{2}, 10 \% \mathrm{CO}_{2}\right)$ for three days. The minimum inhibitory concentration (MIC)

Table 1 Resistant isolates detected by screening agar and E test

\begin{tabular}{lllll}
\hline & Total tested & $\begin{array}{l}\text { Metronidazole } \\
\text { resistant }>8 \mu \mathrm{g} / \mathrm{ml}\end{array}$ & $\begin{array}{l}\text { Clarithromycin } \\
\text { resistant }>2 \mu \mathrm{g} / \mathrm{ml} l\end{array}$ & $\begin{array}{l}\text { Amoxicillin } \\
\text { resistant }>0.5 \mu \mathrm{g} / \mathrm{ml}\end{array}$ \\
\hline E test & 90 & 28 & 4 & 0 \\
Screening agar & 98 & 9 & 0 & 0 \\
Screening agar + E test & 70 & $21^{\star}$ & $4^{\star \star}$ & 0
\end{tabular}

${ }^{\star}$ Detected in five isolates by screening agar.

${ }^{\star \star}$ None detected by screening agar. from the E test was taken as the point at the intersection between the edge of the inhibition zone and the graduations on the $\mathrm{E}$ test strip. Isolates were considered resistant if their MIC was $>8 \mu \mathrm{g} / \mathrm{ml}$ for metronidazole, $>2 \mu \mathrm{g} / \mathrm{ml}$ for clarithromycin, and $>0.5 \mu \mathrm{g} / \mathrm{ml}$ for amoxicillin, as suggested by the European $H$ pylori study group. ${ }^{8}$ In line with $\mathrm{E}$ test reading guides (AB Biodisk; Solna, Sweden), isolated colonies of $H$ pylori growing inside the inhibition zone were noted as resistant mutants (fig $1 \mathrm{~A}$ and $\mathrm{B}$ ). MIC values of three reference strains from the culture collection of the University of Gotenberg (CCUG), sent by the European $H$ pylori study group, were determined by $\mathrm{E}$ test, using the same method described above, for amoxicillin, clarithromycin, and metronidazole on three separate occasions (at the start, halfway, and at the end of the study). The control strains were kept viable by freezing at $-70^{\circ} \mathrm{C}$ in glycerol broth, rather than repeated subculture, to avoid the emergence of possible strain modifications.

\section{Results}

One hundred and twenty eight of the 507 gastric biopsy specimens were positive for $H$ pylori by the biopsy urease test (BUT). ${ }^{10}$ Of these, 123 were culture positive. E-tests were only available for 90 isolates because there was a supply problem with the E test strips during our study. Screening agar results are available for 98 isolates, because the remaining biopsy specimens arrived in the laboratory when the research scientist was absent, so that plates could not be inoculated. Resistance to metronidazole was detected in nine of the 98 isolates by the screening agar (table 1). Using a cut off point of $8 \mu \mathrm{g} / \mathrm{ml}, 28$ of 90 of isolates were metronidazole resistant by the $\mathrm{E}$ test. Seventy isolates had sensitivity test results by both methods. The screening agar failed to detect 16 of the 21 isolates found to be metronidazole resistant by $\mathrm{E}$ test. Two plates were overgrown 
Table 2 E test MICs for CCUG reference strains performed at the beginning, midway, and at the end (runs 1-3) of our study

\begin{tabular}{llll}
\hline QC strain & $\begin{array}{l}\text { Clarithromycin } \\
M I C \mu g / m l\end{array}$ & $\begin{array}{l}\text { Metronidazole } \\
\text { MIC } \mu g / m l\end{array}$ & $\begin{array}{l}\text { Amoxicillin } \\
M I C \mu g / m l\end{array}$ \\
\hline Resistance breakpoint & $>2$ & $>8$ & $>0.5$ \\
CCUG 38770 & & & \\
$\quad$ Expected result & $0.012-0.25$ & $>256$ & 0.015 \\
Run 1 & 0.38 & $>256$ & 0.016 \\
Run 2 & 0.38 & $>256$ & 0.016 \\
Run 3 & 0.25 & $>256$ & 0.016 \\
CCUG 38771 & & & \\
Expected result & 0.03 & 0.5 & $<0.015$ \\
Run 1 & 0.023 & 1 & $<0.016$ \\
Run 2 & 0.023 & 0.19 & $<0.016$ \\
$\quad$ Run 3 & 0.023 & $0.19 \star$ & $<0.016$ \\
CCUG 38772 & & & \\
Expected result & 2 & $32-64$ & $<0.015$ \\
Run 1 & 6 & $>256$ & $<0.016$ \\
Run 2 & 0.75 & 128 & $<0.016$ \\
Run 3 & 6 & 64 & $<0.016$ \\
\hline
\end{tabular}

^Mutant colonies inside the zone.

CCUG, culture collection of the University of Gotenberg; MIC, minimum inhibitory concentration.

with other gastric flora and four isolates only yielded scanty growth on the non-selective agar. Four isolates were clarithromycin resistant (MIC $>2 \mu \mathrm{g} / \mathrm{ml}$ by E test); three of these were resistant subcolonies (fig 1B). The screening agar method failed to detect any clarithromycin resistant isolates. No amoxicillin resistant isolates were detected by $\mathrm{E}$ test or screening agar.

Table 2 shows the reference strain $\mathrm{E}$ test results. Twenty one of the 27 tests were within one dilution of the expected result. Two E test clarithromycin results would have been reported incorrectly as resistant; the expected MIC of CCUG 38772 lay on the breakpoint at $2 \mu \mathrm{g} / \mathrm{ml}$ and our test results were reported at $6 \mu \mathrm{g} / \mathrm{ml}$.

\section{Discussion}

The prevalence of primary metronidazole and clarithromycin resistance in west Gloucestershire is $31 \%$ and $5 \%$, respectively. Amoxicillin resistance was not detected. The prime aim of our project was to determine the reliability of using a screening agar method at primary isolation to detect resistant $H$ pylori. However, we only detected five of the 21 metronidazole resistant and none of the four clarithromycin resistant strains. In their preliminary report Glupczynski et al correctly detected $84 \%$ of metronidazole resistant isolates. ${ }^{7}$ We cannot explain this discrepancy and we have checked that we were using the same antibiotic concentrations and agar. They found that isolates reported falsely as sensitive yielded a very low density culture of $\mathrm{H}$ pylori, or that plates were contaminated by non- $H$ pylori flora. In our study, contamination or low density culture was only recorded in six of the 16 resistant isolates not detected. The use of only one biopsy specimen for culture in our study might have yielded a lighter growth. However, only eight of the 98 cultures on the $H$ pylori selective agar inoculated after the screening plates yielded a lighter growth than the chocolate agar. No growth was recorded in four cases; resistance was recorded in two of these isolates by the $\mathrm{E}$ test. This suggests that using only one biopsy may miss $7-10 \%$ of resistant strains. Glupczynski's group ${ }^{7}$ grind biopsy specimens with an electric grinder; we do not grind our specimens because large studies in our laboratory have not found an improvement in primary isolation rates after grinding. However, it could be that this makes a difference with this screening agar.

The screening agar would be more cost effective if primary screening agar plates were only set up when a BUT inoculated in endoscopy was positive at arrival in the laboratory. This method is now used successfully by Glupczynski et al and may explain why this group's detection rate was much higher. If only specimens with a positive BUT are inoculated, this would reduce the number of plates inoculated by $70 \%$. However, this does rely on staff in the endoscopy department informing staff in the microbiology department of their results.

Oderda et al have presented preliminary results on 45 strains using a multisector screening agar plate containing Dent's selective agar and metronidazole, clarithromycin, or amoxicillin in each sector. Concordance between $\mathrm{E}$ tests and the multisector plates was seen in all 10 metronidazole and 15 of the 16 clarithromycin resistant isolates. ${ }^{11}$ This method looks promising; however, the multisector plates would be costly to produce in terms of staff time and the method needs further evaluation.

The prevalence of metronidazole resistance in west Gloucestershire has risen in the past 10 years from $10 \%$ in $1989,22 \%$ in 1992 , to $31 \%$ in 1999. This is of concern given the widespread use of metronidazole in the treatment of $H$ pylori infection and the lower eradication rate in the presence of metronidazole resistant strains. ${ }^{2}$ Because studies have shown that metronidazole resistance is not reversible, culture for sensitivity testing should now be encouraged and treatment regimens adjusted according to local prevalence data.

Clarithromycin resistance was seen predominantly as subcolonies within the $\mathrm{E}$ test ellipse (fig 1A). These might be difficult to detect on the screening agar plates and may explain why we failed to identify these resistant strains by the screening agar method. Clarithromycin resistance has a greater impact on the outcome of infection than metronidazole resistance, and prevalence is now high in some European countries, especially in children. ${ }^{12}$ The quality control strain CCUG 38772 would have been reported incorrectly as resistant to clarithromycin (reported MIC, $6 \mu \mathrm{g} / \mathrm{ml}$; expected MIC, $2 \mu \mathrm{g} / \mathrm{ml}$ ). When the expected MIC lies on the breakpoint, the results can be unreliable. When the result lies close to the breakpoint it is probably better to report the MIC value. Although amoxicillin resistance was not detected, continuing surveillance for amoxicillin is still necessary because it can cause a small increase in the numbers of resistant subpopulations.

We wish to thank the endoscopy department for their help and support during this study.

$1 \mathrm{NIH}$ consensus conference. Helicobacter pylori in peptic ulcer disease. fAMA 1994;272:65-9. 
2 Penston JG, McColl KEL. Eradication of Helicobacter pylori: an objective assessment of current therapies. $\mathrm{BrF}$ Clin Pharmacol 1997;43:223-43.

3 Xia HX, Keane CT, O'Marain CA. A five year survey of metronidazole and clarithromycin resistance in clinical isolates of Helicobacter pylori [abstract]. Gut 1996;39(suppl 2):A6

Cayla R. How to eradicate Helicobacter pylori. Gastroenterol Clin Biol 1996;20:S119-30.

5 Zwetvan AA, Vandenbrouke-Grauls CMJE, Thijs JC, et al. Stable amoxycillin resistance in Helicobacter pylori. Lancet 1998;352:1595.

6 Banatavala N, Davies GR, Abdi Y, et al. High prevalence of Helicobacter pylori metronidazole resistance in migrants to east London: relation with previous nitroimidazole exposure and gastroduodenal disease. Gut 1994:35:1562-6.

7 Glupczynski Y, Van den Boore C, Goutier S, et al. Evaluation of metronidazole and clarithromycin screening agar plates for in vitro susceptibility testing of Helicobacter pylori [abstract]. Gut 1997;41(suppl 1):A2.
8 Glupczynski G, Mégraud F, Anderson LP, et al. Antibiotic susceptibility of Helicobacter pylori in Europe in 1998: results of the third multi-centre study [abstract]. Gut 1999; 45(suppl 111):A105.

9 Chaves S, Gadanho M, Tenreiro R, et al. Assessment of metronidazole susceptibility in Helicobacter pylori: statistical validation and error rate analysis of breakpoints determined by the disk diffusion test. $\mathcal{F}$ Clin Microbiol 1999;37:1628-31.

10 McNulty CAM, Dent JC, Uff JC, et al. Detection of Campylobacter pylori by the biopsy urease test: an assessment in 1445 patients. Gut 1989;30:1058-62.

11 Oderda G, Rapa A, Ronchi B, et al. A fast and easy to perform test for antibiotic susceptibility of Helicobacter pylori [abstract]. Gut 1999;45(suppl III):A97.

12 Taylor KJ, Edwards-Jones V, Armitage M. Metronidazole sensitivity testing of Helicobacter pylori: the importance of media. Br f Biomed Sci 1998;55:118-22.

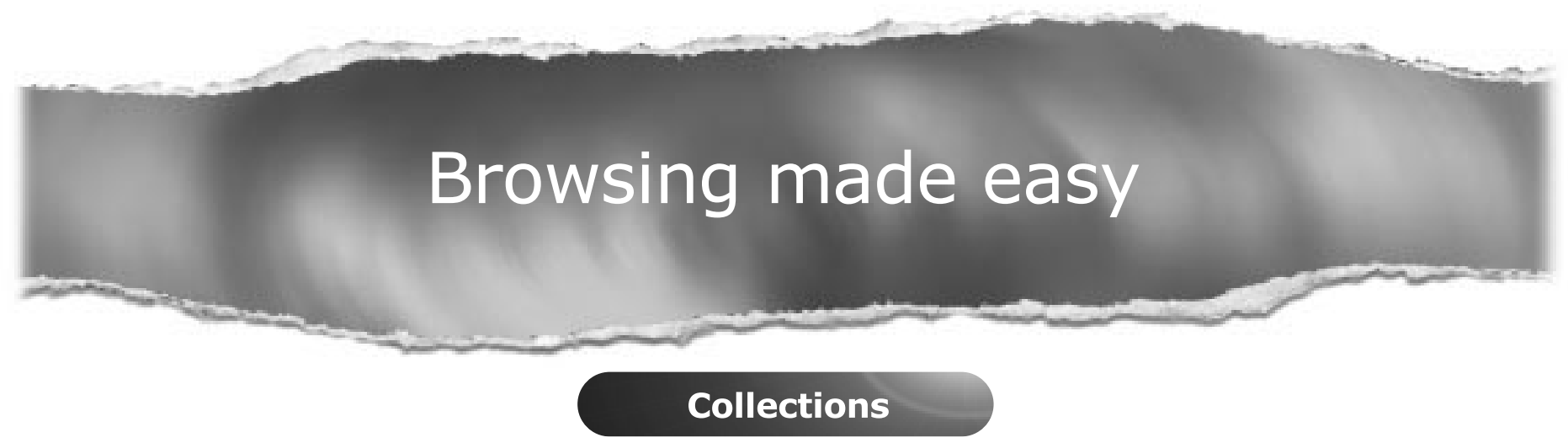

With a single click Collections allows you to find all articles that have been published in your chosen subject. Select from over 200 clinical and non-clinical topic collections and/or cross search other specialist journals, the BMJ and Cochrane Reviews

\section{www.jclinpath.com}

